\title{
Research on financial network big data processing technology based on fireworks algorithm
}

Tao Luo

\begin{abstract}
Big data is a hot issue in both theoretical and practical circles. Although many scholars have analyzed the risk of internal control of venture capital information system from different angles, there is still a lack of research on the risk of internal control of venture capital system under big data environment. Aiming at the concept and characteristics of large data, this paper proposes the research of internal control system of venture capital information system based on large data processing technology. The risk prediction model based on improved quantum support vector machine is used to verify the accuracy of the model. This paper divides the risk critical control process one by one for the hardware, software, personnel, information and operation rules of the venture capital object; probes into the main risks of different control objects in the process of information system construction under the big data environment; and puts forward the corresponding risk management methods of system internal control. Simulation experiments verify the reliability of the model and algorithm.
\end{abstract}

Keywords: Big data, Risk venture, Predict model, Control model, Support vector

\section{Introduction}

With the rapid development of modern science and technology, the amount of data has increased explosively. Traditional computing technology cannot meet the needs of large amount of data calculation and analysis [1-3]. The processing center of software has changed from process control to data value mining, which has become an irresistible trend. Value mining of huge data is widely used in trend prediction, personalized recommendation, and relevance of things. Under the influence of the big data research and development plan put forward by the USA, the big data mining strategy has gradually become an important part of the national core strategy. The UK, Japan, and other countries have followed suit to put forward the big data development strategy. In recent years, China has also issued the big data development strategy $[4,5]$. The concept of large data is first proposed in the fields of physics, meteorology, biology, and other sciences. Facing the problems encountered by a large number of scientific mathematics in acquisition, storage, search, sharing, and analysis,

Correspondence: ytml58@163.com

School of Economics, Xihua University, Chengdu 610039, Sichuan, China some new distributed computing technologies have been studied and developed accordingly [6].

The rapid popularization of the mobile Internet has brought a lot of new changes to society and also made service providers accumulate a large number of individual user data. Big data is a kind of huge information data with high growth rate and various types. It cannot be collected, managed, and analyzed by common software in a certain time scale $[7,8]$. New processing methods and methods must be used to ensure that the company has more correct decision-making power, insight, and process than before [9]. At present, the development of a venture capital enterprise depends more and more on the ability to extract effective information from massive data as the basis for decision-making-level investment activities [10]. Faced with the new era background, venture capital enterprises should speed up the transformation of the inherent thinking, actively embrace new technologies and ideas, and constantly find and solve problems, so as to make it in the future development of an invincible position [11, 12]. Based on the background of big data, venture capital enterprises have welcomed the possibility of greater development than before [13]. 
In the new era, we should pay more attention to the essence of the problem behind the massive data information, and paying more attention to the huge economic benefits brought by the data has become the consensus of major enterprises. The traditional sense of venture capital enterprises in the face of huge amounts of data information, the corresponding level of organization, and management has been unable to meet the market requirements. The changes of the times require that the venture capital enterprises should have more efficient and discriminative data processing ability. The decision-making level should analyze the information obtained from the data processing so as to forecast the market development direction and start the investment business according to the company's investment ability. This will help the enterprises to optimize their business plans and make accurate decisions and to promote the development of enterprises.

\section{Methods}

\subsection{The big data platform for venture capital}

Venture capital is a very complex and related process with many factors (Fig. 1). It is a nonlinear time series with many conditions. It has the characteristics of dynamic, uncertain, nonlinear, and catastrophic. It is necessary to apply large data theory to collect a large number of historical actual venture capital data and knowledge. Through data mining and data analysis, the standard, structure, and standardized description of various factors which have certain impact on risk prediction are established. Accurate risk prediction will help to grasp the state of line investment object in time and provide a reliable basis for investment risk classification. At the same time, investment risk research is a branch of research involving finance, economy, disaster management, and other fields. It is the result of the deepening and refinement of financial system risk theory research and the foundation of establishing risk prevention system.

The data source layer integrates multi-energy data and other internal and external data to form structured data, such as relational database and two-dimensional table. The data warehouse is composed of unstructured data, history, quasi-real time, real-time data, and GIS data.

The business application layer introduces a multi-tenant model, provides various data access control methods and graphical programming framework, and provides decision support for data applications such as intelligent prediction, intelligent investment consulting, and intelligent venture capital control.

The model layer provides some model to deal with the data that come from the data layer and provides some information to the business layer. It is the link bridge between the raw data and intelligent decision.

\subsection{Model construction of big data platform based on Spark}

Spark platform is a new generation of large data analysis framework based on Hadoop MapReduce. It is mainly used to speed up the running and reading and writing of data analysis. It has all the advantages of Hadoop MapReduce. But unlike MapReduce, Spark platform stores the results of calculation directly in memory, so as to make the data analysis faster and faster. The

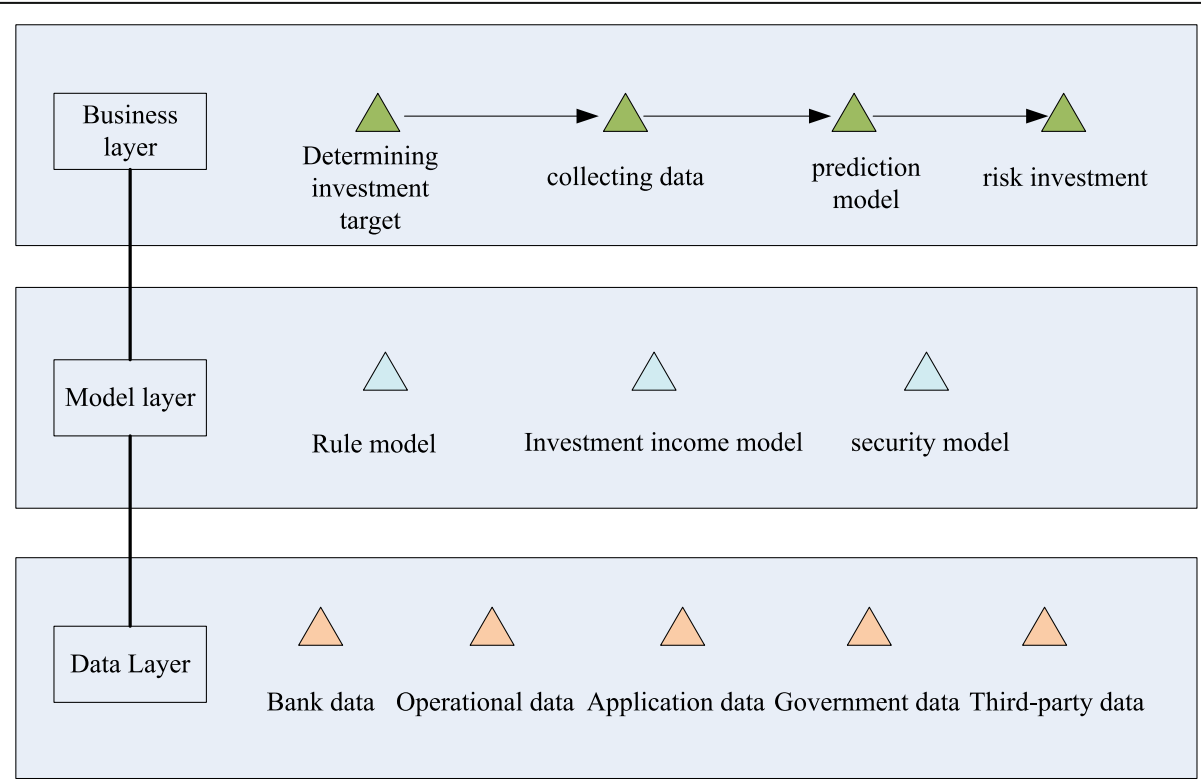

Fig. 1 Big data venture capital platform 
procedure is more efficient. The related tests show that the performance of the Spark platform can exceed 10 100 times of the Hadoop platform. In addition, Spark platform can realize machine learning and interactive analysis of online data. Each thread task can directly fetch the required data from memory, thus realizing the high sharing of data, improving the efficiency of data processing and the speed of algorithm operation. While Hadoop MapReduce needs to write multiple parallel or serial MR tasks when performing data tasks, the data between tasks cannot be shared, resulting in low performance, long analysis time, large memory occupancy, and other issues, and it is only suitable for offline large data analysis. With the rapid development of modern intelligent monitoring equipment, it is very important to mine and apply large data online in real time and efficiently. Therefore, the characteristics of Spark large data platform make it have more advantages in iterative algorithm and interactive data mining algorithm.

The process of venture capital is complex and changeable, and the financial environment changes all the time will produce a huge amount of data. Therefore, establishing the quantitative model of influencing factors, risk prediction model, and risk assessment model based on Spark platform can effectively improve the processing efficiency of massive financial data and reduce the redundancy of information. Thus, it can help to improve the performance of the model, improve the accuracy of the algorithm, and provide a reliable support for the relevant research of venture capital. The related models based on Spark platform are shown in Fig. 2.
As can be seen from Fig. 2, this paper mainly, based on the data warehouse, model base, and knowledge base of venture capital, put the quantitative analysis model of investment factors, risk prediction model, and investment risk assessment model on the Spark platform for data processing and algorithm operation, in order to improve the efficiency of data processing and knowledge base and enhance the performance of the algorithm. Quantitative analysis of influencing factors is an important basis for other models. The study of a large number of historical investment data, such as data classification, bad data processing, missing data supplement, and data dimension unification, can provide good large data preparation for subsequent wind prediction models, investment state risk assessment models, and other operations. In addition, it is necessary to deal with structured data, unstructured data, or mixed data by MapReduce, which is based on the quantification of influencing factors, risk prediction, and investment risk assessment of large-scale data. Parallel computation is realized by developing multiple Spark Streaming processes, parameter optimization, and variable selection, and redundancy is reduced. Information can effectively improve the efficiency and accuracy of the algorithm.

\section{Investment risk prediction model based on Spark big data platform}

3.1 Quantum fireworks optimization algorithm model (QFA)

The fireworks algorithm (FA) is the simulation of the entire fireworks explosion process. When the fireworks explode, a large number of sparks will be generated, and

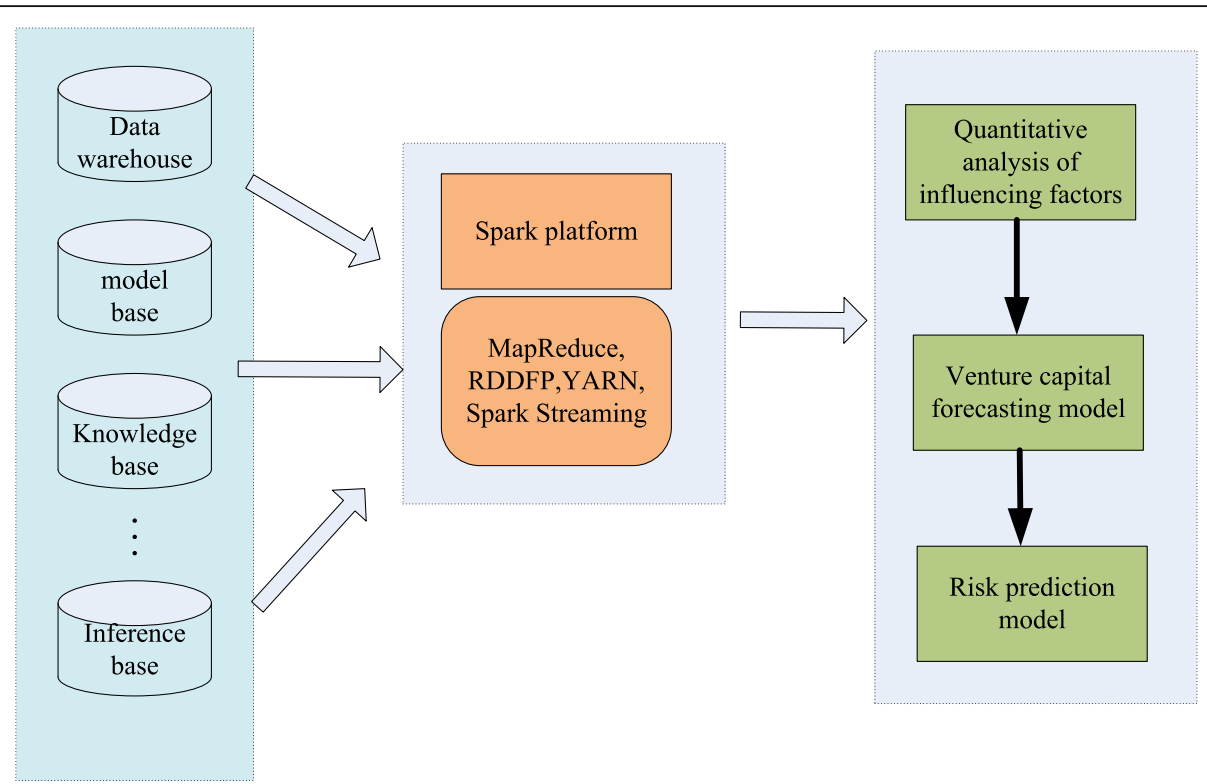

Fig. 2 Model construction of big data platform based on Spark 
the sparks can continue to explode, resulting in beautiful and colorful patterns. In the fireworks algorithm, each firework can be regarded as a feasible solution in the solution space of the optimization problem, so the spark generated by fireworks explosion can be regarded as a process of searching for the optimal solution. In the specific optimization problem, the fireworks algorithm needs to consider the number of sparks produced by each fireworks explosion, the explosion radius, and how to select a set of optimal fireworks and sparks to carry out the next explosion.

In the fireworks algorithm, the most important three components are explosion operator, mutation operator, and selection strategy.

(1) Explosion operator: The number $S_{i}$ and radius $A_{i}$ of sparks produced by each firework $x_{i}(i=1,2 \cdots n)$ are calculated according to the fitness of the fireworks.

$$
\begin{array}{r}
S_{i}=M \times \frac{y_{\max }-f\left(x_{i}\right)+\varepsilon}{\sum_{i=1}^{N}\left(y_{\max }-f\left(x_{i}\right)\right)+\varepsilon} \\
R_{i}=\hat{R} \times \frac{f\left(x_{i}\right)-y_{\min }+\varepsilon}{\sum_{i=1}^{N}\left(f\left(x_{i}\right)-y_{\min }\right)+\varepsilon}
\end{array}
$$

In the above formula, $y_{\max }$ and $y_{\min }$ represent the maximum and minimum fitness values of the current population, $f\left(x_{i}\right)$ is the fitness value of fireworks $x_{i}$, and $M$ is a constant, which is used to adjust the number of sparks produced. $\hat{R}$ is a constant, which is used to adjust the explosion radius of fireworks. $\varepsilon$ is the smallest part of the machine to avoid zero operation.

(2) Mutation operator: The mutation operator is set to increase the diversity of the explosive spark population. The mutation spark in the fireworks algorithm is to generate the Gaussian mutation spark by the Gaussian mutation of the explosive spark. Assuming that fireworks $x_{i}$ is selected for Gaussian mutation, the $k$-dimensional Gaussian mutation operation is $\hat{x}_{i k}=x_{i k} \times e$, where $x_{i k}$ represents $k$-dimensional variant fireworks and $e$ represents $N(1,1)$ Gaussian distribution.

The explosive sparks and mutation sparks generated by the explosive operator and mutation operator in the spark algorithm may exceed the boundaries of the feasible region $\Omega$, which is mapped to a new position by mapping rules. The formula is as follows:

$$
\hat{x}_{i k}=x_{L B, k}+\left|\hat{x}_{i k}\right| \%\left(x_{U B, k}-x_{L B, k}\right)
$$

where $x_{U B, k}$ and $x_{L B, k}$ is the upper and lower bounds of solution space on $k$ dimension respectively.

(3) Selection strategy: In order to transmit the information of the excellent individuals in the group to the next generation, it is necessary to select a certain number of individuals as the fireworks of the next generation from the explosive sparks and variant sparks.

Assuming $K$ candidates and $N$ populations, the individuals with the best fitness in the candidate set will be identified as the next-generation fireworks. The remaining $N-1$ fireworks are selected by probability. For fireworks $x_{i}$, the probability formula for its selection is:

$$
\begin{aligned}
& p\left(x_{i}\right)=\frac{R\left(x_{i}\right)}{\sum_{x_{j} \in K} x_{j}} \\
& R\left(x_{i}\right)=\sum_{x_{j} \in K} d\left(x_{i}-x_{j}\right)=\sum_{x_{j} \in K}\left|x_{i}-x_{j}\right|
\end{aligned}
$$

In the upper form, $R\left(x_{i}\right)$ is the sum of the distances between all the individuals in the current individual candidate set. In the candidate set, if the individual density is high, that is, when there are other candidates around the individual, the probability of the individual being selected will be reduced.

\subsection{Quantum evolutionary algorithm}

The development of quantum mechanics makes quantum computation more and more applied in all aspects. In quantum computation, the representation of quantum states is performed by quantum bits. Usually, the representation of quantum information is performed by the 0 and 1 binary method; besides the " 0 " and " 1 " states which can be in the basic state, the quantum states can also be in the arbitrary linear superposition of the " 0 " and " 1 " states, that is, the two states can be in the same state at the same time. To a great extent, it challenges the representation of classical positions in classical mechanics. The superposition state of quantum state can be expressed by the lower form:

$$
|\psi \geq \alpha| 0>+\left.\beta|1>,| \alpha\right|^{2}+|\beta|^{2}=1
$$

In the above formula, 0 and 1 denote two states of a quantum; $\alpha$ and $\beta$ denote the probability amplitude of a quantum; $|\alpha|^{2}$ denotes the probability of a quantum state at 0 ; and $|\beta|^{2}$ denotes the probability of a quantum state at 1 . Quantum algorithm is updated through quantum revolving door, and its adjustment strategy is as follows:

$$
\left(\begin{array}{c}
\alpha_{i}^{\prime} \\
\beta_{i}^{\prime}
\end{array}\right)=\left(\begin{array}{cc}
\cos (\theta) & -\sin (\theta) \\
\sin (\theta) & \cos (\theta)
\end{array}\right)\left(\begin{array}{c}
\alpha_{i} \\
\beta_{i}
\end{array}\right)
$$

where $\left(\begin{array}{cc}\cos (\theta) & -\sin (\theta) \\ \sin (\theta) & \cos (\theta)\end{array}\right)$ is a quantum revolving gate and $\theta$ is a quantum rotation angle.

Quantum evolutionary algorithm is a kind of algorithm based on probability search. The concept of 
quantum bit and quantum superposition makes quantum evolutionary algorithm have many advantages, such as better population diversity and strong global optimization ability, especially the algorithm has strong robustness and can be well combined with other algorithms.

\subsection{Quantum fireworks algorithm}

\section{(1) Solution space}

In the solution space, $N$ fireworks are randomly generated and their coordinates are initialized. Here, we use the probability amplitude of the quantum bit as the encoding of the current position of the fireworks.

$$
p_{i}=\left[\begin{array}{cccc}
\cos \left(\theta_{i 1}\right) & \cos \left(\theta_{i 2}\right) & \cdots & \cos \left(\theta_{i n}\right) \\
\sin \left(\theta_{i 1}\right) & \sin \left(\theta_{i 2}\right) & \cdots & \sin \left(\theta_{i n}\right)
\end{array}\right]
$$

where $\theta_{i j}=2 \pi \operatorname{rand}()$ is a random number and the $n$ is the number of solution space. $i=1,2, \cdots n$.Therefore, the probability amplitudes of the quantum states of $\mid 0>$ and | 1> are as follows:

$$
\begin{aligned}
& p_{i c}=\left(\begin{array}{llll}
\cos \left(\theta_{i 1}\right) & \cos \left(\theta_{i 2}\right) & \cdots & \cos \left(\theta_{i n}\right)
\end{array}\right) \\
& p_{i s}=\left(\begin{array}{llll}
\sin \left(\theta_{i 1}\right) & \sin \left(\theta_{i 2}\right) & \cdots & \sin \left(\theta_{i n}\right)
\end{array}\right)
\end{aligned}
$$

\section{(2) Solution space transformation}

The searching process of fireworks optimization algorithm is carried out in the actual parameter space $[a, b]$. Since the probabilistic amplitude of fireworks location is within $[0,1]$, it is necessary to decode the probabilistic amplitude into the actual parameter space $[a, b]$, so as to facilitate the searching of fireworks algorithm; if the firework on the individual fireworks $p_{i}$ is $\left[\alpha_{i}^{j}, \beta_{i}^{j}\right]$, the corresponding solution space is changed. The formula is

$$
\begin{array}{ll}
X_{i c}^{j}=0.5\left[b_{i}\left(1+\alpha_{i}^{j}\right)+a_{i}\left(1-\alpha_{i}^{j}\right)\right] & \operatorname{rand}()<p_{i d} \\
X_{i s}^{j}=0.5\left[b_{i}\left(1+\beta_{i}^{j}\right)+a_{i}\left(1-\beta_{i}^{j}\right)\right] & \operatorname{rand}()>p_{i d}
\end{array}
$$

where $\operatorname{rand}()$ is the random number between $[0,1]$; $X_{i c}^{j}$ is the actual parameter value of the position of the $j$ th dimension when the quantum state of the first fireworks individual is $\mid 0>; X_{i s}^{j}$ is the actual parameter value of the position of the $j$ th dimension when the quantum state of the second fireworks individual is $\mid 1>a_{i}$ and $b_{i}$ are the upper and lower limits of individual $p_{i}$ search range respectively.

(3) Fitness value $f\left(x_{i}\right)$ of each firework was calculated, and the number of sparks produced by the explosion radius $R_{i}$ of each firework was calculated as formulas (1 and 2)

(4) Fireworks individual location update

In this paper, the quantum revolving gate is used to replace the uniformly distributed $U(a, b)$ to update the position of fireworks.

$$
\left(\begin{array}{c}
\alpha_{j d}^{k+1} \\
\beta_{j d}^{k+1}
\end{array}\right)=\left(\begin{array}{cc}
\cos \left(\theta_{j d}^{k+1}\right) & -\sin \left(\theta_{j d}^{k+1}\right) \\
\sin \left(\theta_{j d}^{k+1}\right) & \cos \left(\theta_{j d}^{k+1}\right)
\end{array}\right)\left(\begin{array}{c}
\alpha_{j d}^{k} \\
\beta_{j d}^{k}
\end{array}\right)
$$

$\alpha_{j d}^{k+1}, \beta_{j d}^{k+1}$ is the probability amplitude of the $j$-firework $d$-dimension space in the first $k+1$ iteration. $\theta_{j d}^{k+1}$ is the rotation angle. In addition, to adapt to the operation mechanism of fireworks algorithm, the updated probability amplitudes $\alpha_{j d}^{k+1}$ and $\beta_{j d}^{k+1}$ are transformed into solution spaces.

$$
\begin{aligned}
& X_{i c}^{j}=0.5\left[b_{i}\left(1+\alpha_{j d}^{k+1}\right)+a_{i}\left(1-\alpha_{j d}^{k+1}\right)\right] \quad \operatorname{rand}()<p_{i d} \\
& X_{i s}^{j}=0.5\left[b_{i}\left(1+\beta_{j d}^{k+1}\right)+a_{i}\left(1-\beta_{j d}^{k+1}\right)\right] \quad \operatorname{rand}()>p_{i d}
\end{aligned}
$$

And then calculate the position offset:

$$
\begin{aligned}
& h_{j c}^{d}=R_{i} \times X_{j c}^{d} \\
& h_{j s}^{d}=R_{i} \times X_{j s}^{d}
\end{aligned}
$$

In cross-boundary detection, if the spark of explosion exceeds the boundary of feasible region, the particle position is updated according to formula (3):

\section{(5) Individual mutation operation}

The main reason for premature convergence and local optimum of fireworks population is that the diversity of the population is lost in the process of searching. In quantum fireworks algorithm, quantum mutation is used to replace the Gaussian mutation in the original algorithm to increase the diversity of the population, thus effectively avoiding the abovementioned problems; random selecting of fireworks $x_{i}$, quantum variation spark $\hat{M}$ is generated. Its operation is shown in the following formula:

$$
\left[\begin{array}{l}
01 \\
10
\end{array}\right]\left[\begin{array}{c}
\cos \left(\theta_{i j}\right) \\
\sin \left(\theta_{i j}\right)
\end{array}\right]=\left[\begin{array}{c}
\cos \left(\frac{\pi}{2}-\theta_{i j}\right) \\
\sin \left(\frac{\pi}{2}-\theta_{i j}\right)
\end{array}\right]
$$

The probability of individual variation of fireworks is $p_{m}$, and rand () is a random number between 1,0 ; if 
$\operatorname{rand}()<p_{m}$, the above formula is used to mutate the individual fireworks, change the probability amplitude in the quantum bit, and finally transform the mutated individual fireworks into a solution space and save it to the mutated spark population.

$$
\begin{array}{ll}
X_{j c}^{d}=\frac{1}{2}\left[b_{j}\left(1+\cos \left(\frac{\pi}{2}-\theta_{i j}\right)\right)+a_{j}\left(1-\cos \left(\frac{\pi}{2}-\theta_{i j}\right)\right)\right] \quad \operatorname{rand}()<p_{i d} \\
X_{j s}^{d}=\frac{1}{2}\left[b_{j}\left(1+\sin \left(\frac{\pi}{2}-\theta_{i j}\right)\right)+a_{j}\left(1-\sin \left(\frac{\pi}{2}-\theta_{i j}\right)\right)\right] \quad \operatorname{rand}()>p_{i d}
\end{array}
$$

(6) Using the probability selection formula from fireworks, explosive sparks and Gaussian variant spark population $p\left(x_{i}\right)$ selects $N$ individuals as fireworks for next-generation iteration calculation

\subsection{QFA-SVM risk prediction under Spark big data platform}

DWT-IR feature extraction and QFA-SVM model risk prediction process based on Spark platform is shown in Fig. 3. As can be seen from the graph, the risk prediction system proposed in this paper mainly includes three Spark working groups: the first working group is mainly based on discrete wavelet inconsistency feature selection (part 1), the second working group is sample training (part 2), and the third working group is mainly based on QFA-SVM risk prediction (part 3). When the feature subset cannot satisfy the stopping condition of the algorithm, it will return to the program and continue to loop until the desired accuracy is achieved and the optimal feature subset is output. Therefore, in the risk prediction system proposed in this paper, the first part is to find the optimal feature subset and the optimal regression model parameters by iterative calculation; the second part is to calculate the prediction accuracy of training samples in each iteration, so that the fitness function value can be calculated; and the third part will be that the optimal feature subset and parameters obtained from the above two parts are used to predict the risk probability values of the test samples through the retraining of the regression model.

\section{Experience}

\subsection{Prediction of big data selection and pre-processing 4.1.1 Discrete wavelet transform}

Discrete wavelet transform (DWT) has been widely used in digital signal processing, petroleum exploration, earthquake prediction, medical fault diagnosis, coding theory, quantum physics, and probability theory. Various fast Fourier transform (FFT) and DWT algorithms have emerged constantly and become the most active research field in numerical algebra. Their significance is far beyond the scope of algorithm research, thus opening up a new situation for many scientific and technological fields. Continuous wavelet transform is often redundant. In order to solve this problem, we use discrete wavelet transform to deal with practical problems, that is, discrimination of wavelet basis functions. This discrimination method is not for the time $t$, but for the scaling factor and translation factor in the wavelet basis function. Usually, the wavelet basis function can be represented by the following expression:

$$
\psi_{a, t}(t)=a^{-\frac{1}{2}} \psi\left(\frac{t-\tau}{a}\right) \quad a>0, \tau \in R
$$

where $a$ represents the wavelet scaling factor and $\tau$ is the wavelet shift factor. The discrete wavelet coefficient $\mathrm{WT}_{f}$ can be used to construct function $f(t)$, which is as follows:

$$
f(t)=\frac{1}{A} \sum \mathrm{WT}_{f}(j, n) \psi_{j, n}(t)
$$

where $A$ is a constant, $\mathrm{WT}_{f}(j, n)=\int_{\mathrm{R}} f(t) \psi_{j, n}(t) d t, \mathrm{WT}_{f}(j$ $, n)=2^{-\frac{j}{2}} \psi\left(\frac{t}{2^{\prime}}-n \cdot T_{s}\right)$.

The process of wavelet transform can be expressed as a decomposition tree composed of low-frequency signal and high-frequency signal. In the wavelet decomposition tree, the decomposition process of the signal can be iterated, the multi-level decomposition can be carried out, the low-frequency signal can be decomposed, more low-frequency signal can be obtained, the high-frequency signal can be decomposed, and more resolution can be obtained. In practice, the decomposition frequency of low-frequency signal and high-frequency signal is determined by the coefficient $\mathrm{WT}_{f}$.

\subsubsection{Data inconsistency rate calculation (IR)}

The purpose of feature selection under financial and other historical data is to distinguish the data features with the strongest correlation with risk data, so that the input value of the risk prediction model has a strong pertinence; reduce data redundancy; and help to improve the accuracy of risk prediction to the greatest extent. Data inconsistency rate can accurately describe the discrete characteristics of input features, different feature attributes can get different partition patterns, different partition patterns can get different frequency distribution, the calculation of inconsistency rate can just be used to distinguish the ability of data categories, and the greater the data inconsistency rate, indicating the feature vector, the worse the classification ability.

Feature selection based on data inconsistency is to select the strongest feature subset from all the features of 


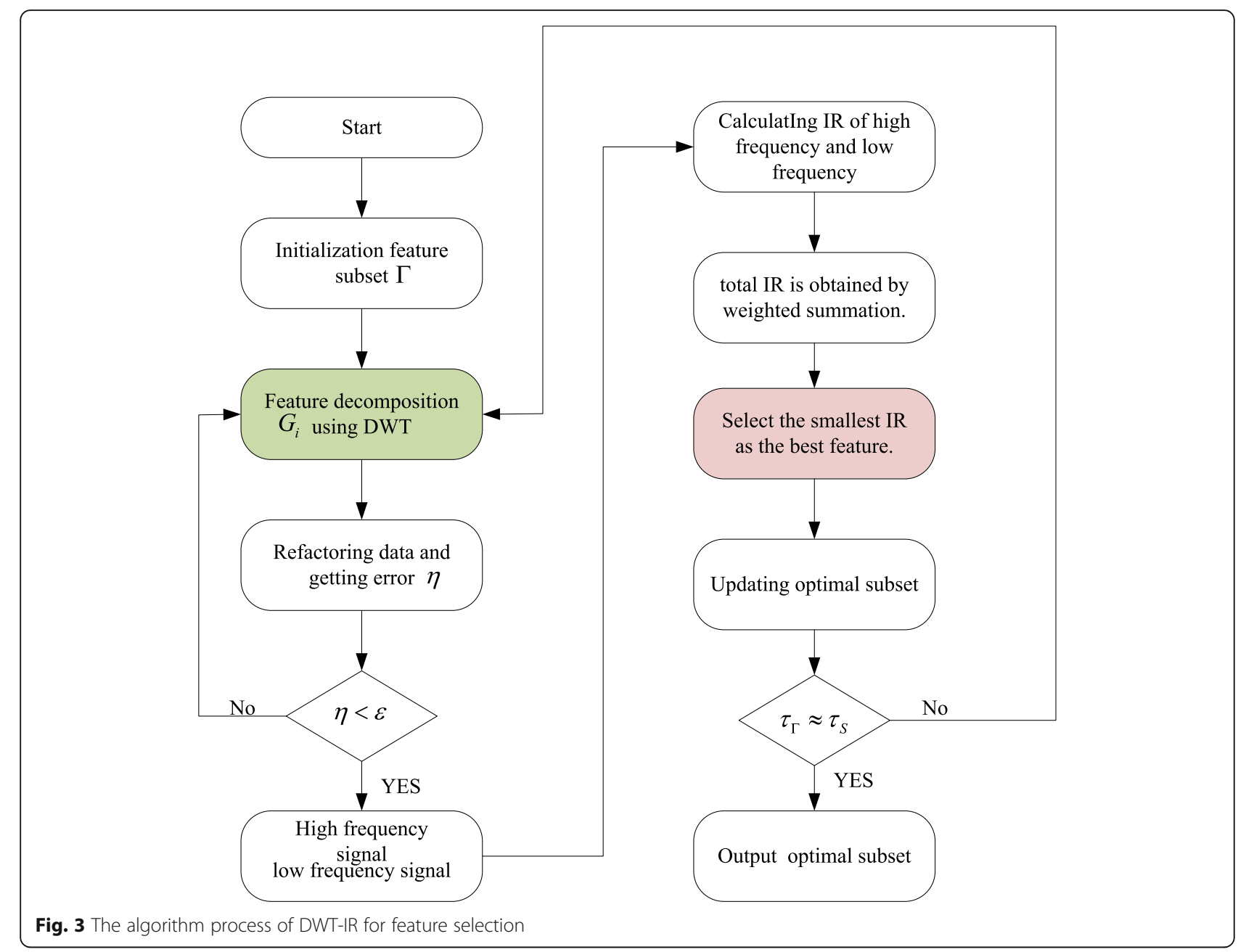

the sample data set and select the feature with the least data inconsistency to maximize the features of the sample data set, which can reduce the redundancy caused by information duplication.

\subsubsection{DWT-IR feature extraction model}

The key of feature extraction method based on the inconsistency rate of discrete wavelet is to calculate the inconsistency rate of different signal groups accurately and judge them accurately. Through the discrete decomposition of the ice-covered feature data, the change of the data characteristics can be reflected more directly. The redundancy of the data can be judged intuitively by the calculation of the inconsistency rate, and the features can be extracted from the large-scale ice-covered data quickly and effectively, thus helping the model to proceed in a favorable direction. The algorithm flow based on DWT-IR feature selection is shown in Fig. 3.

As is shown in Fig. 3, the core of the DWT-IR is how to refractor the data and select the smallest IR as the best feature.

\subsection{Feature extraction based on DWT-IR}

The main purpose of feature selection is to find the optimal subset from a specific problem to describe the accuracy of the prediction system, so as to avoid the redundancy of information caused by too many input vectors, resulting in the decline of prediction accuracy and prediction inaccuracy. Therefore, choosing the appropriate fitness function in the risk prediction system is of great help to determine the optimal feature subset. In this paper, the fitness function based on the double factors of prediction accuracy and feature selection quantity is established:

$$
\text { fitness }\left(x_{i}\right)=-\left(a \times r\left(x_{i}\right)+b \times \frac{1}{\operatorname{Numfeature}\left(x_{i}\right)}\right)
$$

where in the formula, $r\left(x_{i}\right)$ represents the accuracy of risk prediction for each iteration, Numfeature $\left(x_{i}\right)$ is the optimal number of features selected for each iteration, and $a$ and $b$ are constants between $[0,1]$. It can be 
inferred from the formula that the fitness function value will be higher when the risk prediction accuracy is higher and the number of features is less.

Figure 4 is an iterative process chart of the discrete wavelet inconsistency model for feature extraction of training samples.

The fitness curve shown in the figure above describes the fitness function values calculated during each iteration; the accuracy curve describes the predictive accuracy values of the training samples for different iterations; the number of features reduced is the number of features eliminated in the convergence process; and the number of choices represents the convergence of the algorithm. The optimal number of features obtained from the DWT-IR model is calculated. As can be seen from Fig. 4, the algorithm converges when the number of iterations is 46 , and the optimal fitness function is -0.93 . At the 46th iteration, the prediction accuracy of the training samples is $98.8 \%$, which shows that the fitting ability of the model is strengthened by the learning and training of the algorithm, and the prediction accuracy of the training samples is the highest. In addition, when the algorithm runs to the 46th time, the number of selected features tends to be stable. It can be seen that the algorithm eliminates 15 redundant features from 20 candidate features.

\subsection{Risk model prediction based on QFA-SVM}

After obtaining all the optimal features of the sample data, the input vector is brought into the QFA-SVM model for training and testing. In order to ensure the accuracy and accuracy of the SVM model, the wavelet kernel function is selected as the kernel function of the
QFA-SVM regression model, and the important parameters of the model are optimized by the QFA algorithm.

The parameters of the QFA model are as follows: the maximum number of iterations is Maxgen $=500$, the population number is PopNum $=30$, the spark number is constant $M=100$, the explosion radius is constant $R=150$, and the upper and lower bounds of the individual search space of fireworks are $V_{\text {top }}=512$ and $V_{\text {down }}=-512$ respectively. The QFA-SVM model parameters calculated by running program are $\gamma=4756.42$ and $\sigma=8812.18$ respectively.

In order to prove the prediction performance of the proposed ice thickness prediction model, support vector machine (SVM), BP neural network (BPNN), and multi-variable linear regression model (MLRM) are selected to predict the risk of this case. Then, the prediction results of the four models are evaluated and analyzed. In a single SVM prediction model, the selection of its parameters is as follows: $C=913.10, \varepsilon=$ 0012.0, $\sigma=2.4532$. In the BPNN icing prediction model, the number of neurons in the input layer, hidden layer, and output layer is set to 5,7 , and 1 respectively; the neuron activation function is chosen to be sigmoid function; the maximum allowable error of model training is 0.001 , and the maximum number of iterations is set to 5000. This paper selects relative error (relative error, RE) and average absolute percentage error, and the absolute percentage error (MAPE) and root mean square relative error (RMSE) are used as the evaluation indexes of the prediction results (Table 1). The formulas are as follows:

$$
\mathrm{RE}=\frac{y(i)-\hat{y}(i)}{y(i)} \times 100 \%
$$
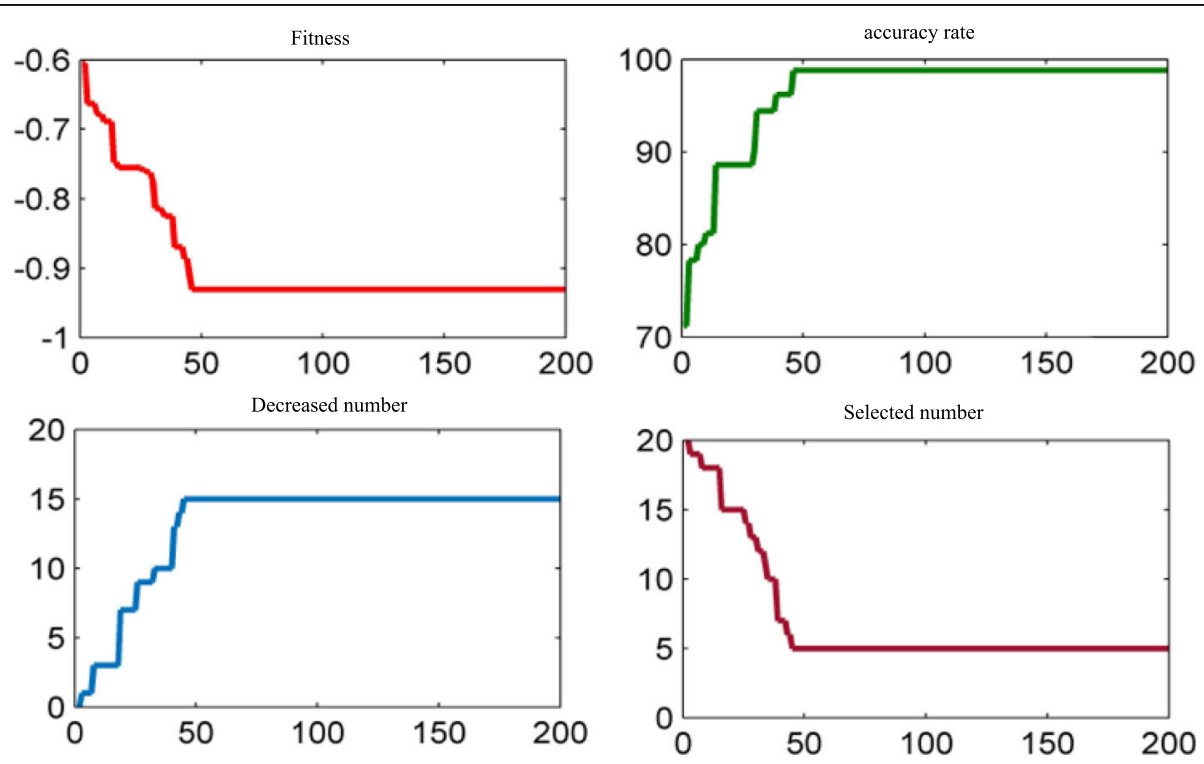

Fig. 4 The curve of convergence for feature selection 
Table 1 Part of the forecasting values and relative errors of each model

\begin{tabular}{|c|c|c|c|c|c|c|c|c|c|}
\hline & \multirow{2}{*}{$\begin{array}{l}\text { Real } \\
\text { data } \\
(\%)\end{array}$} & \multicolumn{2}{|l|}{ QFA-SVM } & \multicolumn{2}{|l|}{ SVM } & \multicolumn{2}{|l|}{ BPNN } & \multicolumn{2}{|l|}{ MLRM } \\
\hline & & Prediction & Error\% & Prediction & Error\% & Prediction & Error\% & Prediction & Error\% \\
\hline 1 & 11.67 & 11.84 & 1.46 & 11.29 & -3.26 & 11.21 & -3.94 & 11.28 & -3.34 \\
\hline 2 & 12.49 & 12.23 & -2.08 & 11.89 & -4.80 & 11.97 & -4.16 & 13.2 & 5.68 \\
\hline 3 & 11.67 & 11.42 & -2.14 & 11.39 & -2.40 & 12.02 & 3.00 & 10.94 & -6.26 \\
\hline 4 & 10.65 & 10.83 & 1.69 & 10.13 & -4.88 & 11.31 & 6.20 & 11.03 & 3.57 \\
\hline 5 & 9.8 & 9.98 & 1.84 & 10.19 & 3.98 & 10.32 & 5.31 & 9.23 & -5.82 \\
\hline 6 & 10.67 & 10.44 & -2.16 & 10.29 & -3.54 & 10.16 & -4.78 & 11.29 & 5.81 \\
\hline 7 & 10.14 & 10.42 & 2.76 & 10.62 & 4.73 & 10.46 & 3.16 & 10.84 & 6.90 \\
\hline 8 & 11.29 & 11.46 & 1.51 & 10.89 & -3.54 & 11.8 & 4.52 & 10.76 & -4.74 \\
\hline 9 & 12.01 & 12.24 & 1.92 & 11.6 & -3.41 & 12.42 & 3.41 & 11.26 & -6.24 \\
\hline 10 & 12.39 & 12.62 & 1.86 & 11.84 & -4.44 & 12.97 & 4.68 & 11.64 & -6.05 \\
\hline MAPE & & & 2.02 & & 3.85 & & 4.41 & & 5.28 \\
\hline RMSE & & & 2.05 & & 3.94 & & 4.50 & & 5.38 \\
\hline
\end{tabular}

$$
\begin{aligned}
\text { MAPE } & =\frac{1}{N} \sum_{i=1}^{N}\left|\frac{y(i)-\hat{y}(i)}{y(i)}\right| \times 100 \% \\
\text { MAPE } & =\sqrt{\frac{1}{N} \sum_{i=1}^{N}\left|\frac{y(i)-\hat{y}(i)}{y(i)}\right|^{2}}
\end{aligned}
$$

Through program operation calculation, the prediction results of the total icing thickness of the four models are shown in Fig. 5.

First, the maximum and minimum relative distance between the original risk and the predicted value can be seen from Fig. 5. In the MLRM model, the maximum relative distance is 0.77 and the minimum relative distance is 0.25 . In the BPNN model, the maximum and minimum relative distances are 0.56 and 0.16 respectively. The two values of BPNN are less than MLRM, indicating that the prediction accuracy of BPNN is higher than that of MLRM. In the SVM model, the maximum and minimum relative distances are 0.47 and 0.11 respectively. The deviation between the maximum and minimum relative distances is 0.36 , which is smaller than that of the BPNN and MLRM models. It shows that the stability of the SVM icing prediction model is higher than that of the BPNN and MLRM. In the proposed QFA-SVM icing prediction model, the maximum relative distance is only 0.2 , the minimum relative distance is 0.064 , and the deviation between the maximum and minimum relative distances is only 0.136 . Compared

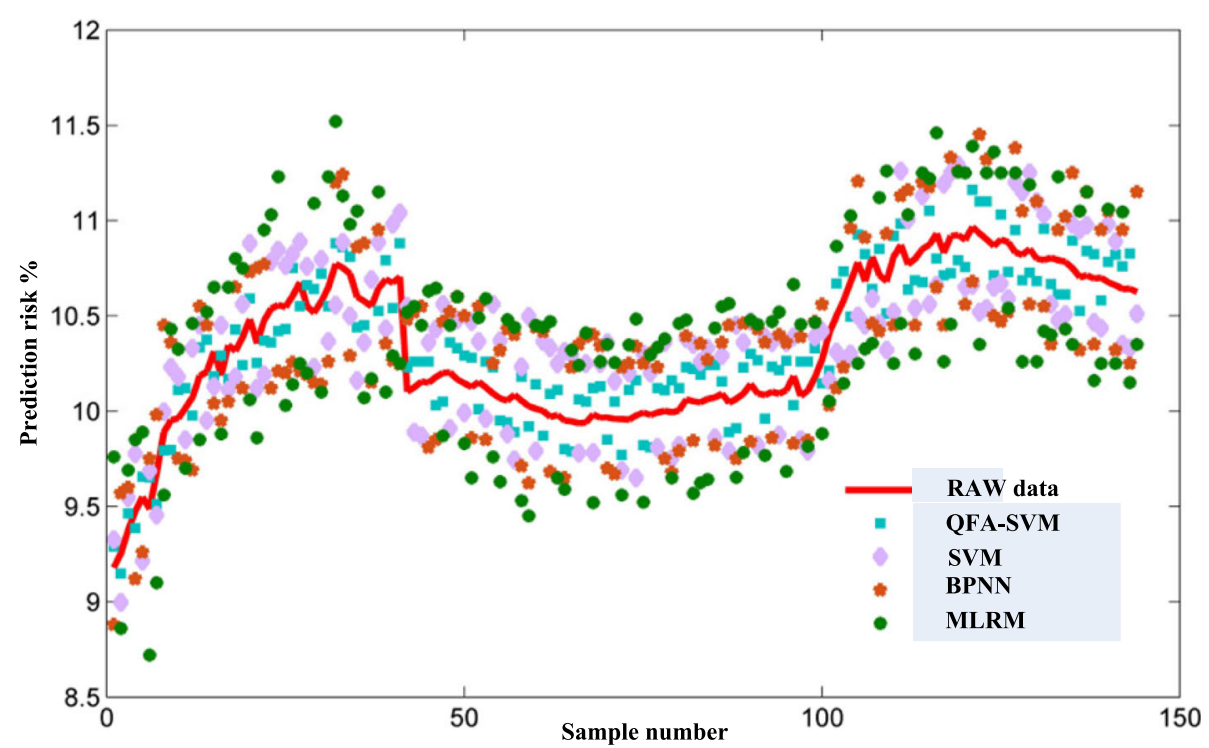

Fig. 5 The prediction risk of the QFA-SVM 
Table 2 The risk grade of the venture

\begin{tabular}{llll}
\hline Risk probability (\%) & Risk grade & Degree of risk & Color \\
\hline $0-5$ & 1 & LR & Gray (g) \\
$5-10$ & 2 & NR & Blue (b) \\
$10-20$ & 3 & SR & Yellow (y) \\
$20-30$ & 4 & SR & Orange (o) \\
$30+$ & 5 & HR & Red (r) \\
\hline
\end{tabular}

with the other three prediction models, the three values of the QFA-SVM model are smaller; it shows that the proposed icing prediction model has higher values. It has prediction accuracy and higher prediction stability.

\subsection{Dynamic assessment of risk status}

According to the possible influence degree, influence range, and development trend of venture capital events, the risk grade of investment state is divided into five grades according to the ice thickness range. Grade I (mild), grade II (general), grade III (moderate), grade IV (severe), and grade V (very serious) are represented in gray, blue, yellow, orange, and red in turn, as shown in Table 2.

Usually, the state parameters of investment risk assessment will change with time, and the investment data have dynamic changes. If the state parameters of large amount of data are directly input into the DBN-ANFIS model, the operation efficiency of the risk assessment model will be slow, and the state evaluation will be inaccurate. Therefore, in order to improve the data processing efficiency and model accuracy, this paper puts the DBN-ANFIS model on the Spark large data platform for the state risk assessment of large amount of data.

The convergence times of the DBN-ANFIS model, ANFIS model, and BPNN model are 354, 417, and 442 respectively, and the model precision is $0.0855,0.1132$, and 0.1285 respectively. The convergence times and model precision of the DBN-ANFIS model are better than those of the ANFIS and BPNN model, which shows that the convergence speed and global search ability of the DBN-ANFIS model are important. It is better than the other two models. Through the improvement of dynamic Bayesian reasoning, the global fitting ability of the ANFIS model is improved, and the algorithm converges quickly and achieves the expected precision in the training of samples, thus enhancing the robustness of the ANFIS model.

As shown in Table 3, the MAPE value of the DBN-ANFIS model is $2.74 \%$, less than $4.69 \%$ and $4.98 \%$ of the ANFIS model and BPNN model; in addition, the MSE value of the DBN-ANFIS model is 0.17 , also less than the ANFIS model (0.68) and BPNN model (0.74); the smaller the MSE value, the higher the prediction accuracy of the DBN-ANFIS model for output value 1Y. And the stability is stronger. In addition, the recognition rate of the DBN-ANFIS model for risk degree and color identification was $97.31 \%$, and the number of identification errors was 7, including 1 LR sample, 5 NR sample, and $1 \mathrm{MR}$ sample; the recognition rate of the ANFIS model was $92.69 \%$, and the number of identification errors was 19, including 1 LR sample, 9 NR sample, and 1 MR sample. The recognition rate of the BPNN model is $91.92 \%$, and the number of recognition errors is 21 , including $1 \mathrm{NR}$ sample, $5 \mathrm{MR}$ sample, $10 \mathrm{SR}$ sample, and 5 HR sample. The above data show that the DBN-ANFIS model is more accurate than the ANFIS and BPNN models in identifying the degree of risk and color identification, especially for SR and HR levels.

\section{Results and discussion}

Financial network based on fireworks algorithm big data processing technology research, mainly discusses the

Table 3 The MAPE value of the DBN-ANFIS, ANFIS, and BPNN models

\begin{tabular}{|c|c|c|c|c|c|c|c|}
\hline & \multirow{2}{*}{$\begin{array}{l}\text { Real } \\
\text { data } \\
(\%)\end{array}$} & \multicolumn{2}{|c|}{ DBN-ANFIS } & \multicolumn{2}{|l|}{ ANFIS } & \multicolumn{2}{|l|}{ BPNN } \\
\hline & & Prediction & Color & Prediction & Color & Prediction & Color \\
\hline 1 & 5.26 & 4.95 & g & 4.91 & g & 4.90 & Gray (g) \\
\hline 2 & 9.61 & 10.1 & y & 10.22 & y & 9.01 & Blue (b) \\
\hline 3 & 13.66 & 13.41 & y & 14.18 & y & 12.44 & Yellow (y) \\
\hline 4 & 15.38 & 15.67 & y & 15.86 & y & 14.76 & Yellow (y) \\
\hline 5 & 20.71 & 20.20 & 0 & 20.13 & o & 19.11 & Yellow (y) \\
\hline 6 & 18.42 & 18.65 & y & 19.65 & y & 20.06 & Orange (o) \\
\hline 7 & 30.47 & 31.15 & $r$ & 29.36 & o & 29.33 & Orange (o) \\
\hline 8 & 29.12 & 28.66 & o & 31.07 & r & 27.77 & Orange (o) \\
\hline 9 & 19.45 & 19.68 & y & 21.38 & o & 17.73 & Yellow (y) \\
\hline 10 & 19.92 & 20.16 & o & 20.68 & o & 19.20 & Yellow (y) \\
\hline Accuracy rate & & & $97.31 \%$ & & $92.69 \%$ & & 4.41 \\
\hline
\end{tabular}


advancement of fireworks algorithm, the fireworks algorithm has the ability of intelligent processing, at the same time has a comprehensive processing of a large number of statistical data analysis ability, in the process of a variety of data fusion analysis play an important role, for the processing of financial data have very good reference.

\section{Abbreviations}

DWT: Discrete wavelet transform; FFT: Various fast Fourier transform; MLRM: Multi-factor linear regression model; MR: Magnetic resonance

\section{Funding}

Youth fund for the humanities and social sciences research of China's Ministry of Education. Research on the incentive mechanism of economic development of China's model_-based on the perspective of stochastic dynamic optimization. 15XJC630005.

\section{Availability of data and materials}

The data sets used and/or analyzed during the current study are available from the corresponding author on reasonable request.

\section{Author's information}

Tao Luo, Doctor of Finance, Assistant Professor, graduated from Southwestern University of Finance and Economics in 2013 and worked in Xihua University. His research interests include real option, game, and corporate finance.

\section{Competing interests}

The author declares that he has no competing interests.

\section{Publisher's Note}

Springer Nature remains neutral with regard to jurisdictional claims in published maps and institutional affiliations.

Received: 8 January 2019 Accepted: 18 April 2019

Published online: 17 May 2019

\section{References}

1. A. Sahaym, S.Y. Cho, K.K. Sang, et al., Mixed blessings: how top management team heterogeneity and governance structure influence the use of corporate venture capital by post-IPO firms. J. Bus. Res. 69(3), 1208-1218 (2016)

2. S. Anokhin, S. Peck, J. Wincent, Corporate venture capital: the role of governance factors. J. Bus. Res. 69(11), 4744-4749 (2016)

3. T.L. Galloway, D.R. Miller, A. Sahaym, et al., Exploring the innovation strategies of young firms: corporate venture capital and venture capital impact on alliance innovation strategy. J. Bus. Res. 71-79 (2016)

4. T. Minola, S. Vismara, D. Hahn, Screening model for the support of governmental venture capital. J. Technol. Transfer. 1(1), 1-19 (2016)

5. Madni, S.H.H., Latiff, M.S.A., Coulibaly, Y., Abdulhamid, S.I.M.: An appraisal of meta-heuristic resource allocation techniques for laaS cloud. Indian J. Sci. Technol. 9(4), 1-14 (2016)

6. S.U. Lee, G. Park, J. Kang, The double-edged effects of the corporate venture capital unit's structural autonomy on corporate investors' explorative and exploitative innovation. J. Bus. Res. 88, 141-149 (2018)

7. M. Scarlata, J. Walske, A. Zacharakis, Ingredients matter: how the human capital of philanthropic and traditional venture capital differs. J. Bus. Ethics 145(3), 1-13 (2017)

8. C. Bock, A. Huber, S. Jarchow, Growth factors of research-based spin-offs and the role of venture capital investing. J. Technol. Transfer. 2017(1), 1-35 (2017)

9. M.G. Colombo, D.J. Cumming, S. Vismara, Governmental venture capital for innovative young firms. J. Technol. Transfer. 41(1), 10-24 (2016)

10. N. Wasserman, Revisiting the strategy, structure, and performance paradigm: the case of venture capital. Organ. Sci. 19(2), 241-259 (2016)

11. A. Jolink, E. Niesten, The impact of venture capital on governance decisions in collaborations with start-ups. Small Bus. Econ. 47(2), 1-14 (2016)

12. C. Weber, B. Bauke, V. Raibulet, An empirical test of the relational view in the context of corporate venture capital. Strateg. Entrep. J. 10(3), 274-299 (2016)

13. A. Buchner, S. Espenlaub, A. Khurshed, et al., Cross-border venture capital investments: the impact of foreignness on returns. J. Int. Bus. Stud. 11(6), 130 (2018)

\section{Submit your manuscript to a SpringerOpen ${ }^{\circ}$ journal and benefit from:}

- Convenient online submission

- Rigorous peer review

- Open access: articles freely available online

- High visibility within the field

- Retaining the copyright to your article

Submit your next manuscript at $\boldsymbol{\nabla}$ springeropen.com 